\title{
DIE MARITIME ORTUNG BEI EINIGEN STAMMEN IN NORDOST-NEUGUINEA
}

\author{
Von Georg HöltKer
}

\begin{abstract}
Ortung ist Orientierung im Raum. In diesem Sinne ist "Ortung" als terminus technicus bei Geographen, Ethnologen und Prähistorikern in Gebrauch. Bei dem Wort "Orientierung" denkt man zunächst an ein Sichzurechtfinden im Raum nach den Sonnenständen, insbesondere nach dem Osten, dem "Orient», der Gegend des Sonnenaufganges. Dagegen ist das Wort «Ortung» mit dieser Etymologie nicht belastet; es ist wurzelhaft neutraler und darum allgemein besser zu gebrauchen. Es gibt nämlich viele Naturvölker und andere, die sich im Raum nicht nach der Sonne orientieren, sondern nach dem Mond oder nach den Plejaden oder nach der Milchstraße oder nach Winden, Bergen, Flüssen usw. Verschiedene Völker und Stämme orientieren sich ausschließlich oder vorwiegend nach dem Meer. Ich möchte die Orientierung nach der Sonne «solare Ortung», die nach dem Meer «maritime Ortung» nennen. Im folgenden wird von dieser maritimen Ortung die Rede sein.
\end{abstract}

\section{DAS TATSACHENMATERIAL}

Die Tatsachen, die ich hier in einer Erstveröffentlichung vorlege, sind originaldokumentarisches Material meiner Feldforschung, gesammelt an Ort und Stelle während meiner ethnologischen Neuguinea-Expedition 1936-1939. Gewährsleute waren alte und ältere Eingeborne aus den betreffenden Stämmen. Als Dolmetscher bzw. Experten in der jeweiligen Lokalsprache, wenn bei der Vielheit der Idiome meine eigenen Sprachkenntnisse nicht reichten, waren mir die Missionare SVD. behilflich, besonders J. Schebesta, K. Böhm und Joseph MuCh, deren ich auch an dieser Stelle dankbar gedenke.

Mein Forschungsfeld lag zwischen der Sepikmündung und dem Hatzfeldthafen an der NO-Küste Neuguineas, insbesondere im sogenannten Bogiabezirk und auf den vorgelagerten Inseln. Die genauere Lokalisierung soll nachher bei der Behandlung der einzelnen Stämme angegeben werden. Aus Gründen größerer Übersichtlichkeit, aber auch wegen der kulturellen Verschiedenheiten, empfiehlt es sich, die einzelnen Stammesgruppen getrennt aufzuführen. Sprachlich gehören diese Eingebornen teils zu den melanesisch-, teils zu den papuanischsprechenden Völkern. Teils sind sie typische Insulaner, teils ebenso typische Festlandbewohner, teils aber auch auf dem Festland siedelnde ehemalige Inselleute. Zur eigentlichen Inlandbevölkerung gehört jedoch keiner dieser Stämme. Alle betreiben einen ziemlich ausgedehnten Ackerbau, ergänzen aber ihre pflanzliche Nahrung (besonders Taro und Yams) durch Jagd und Fischfang. Sago spielt nur bei den Nubia-Awar eine nennenswerte, wenn auch im ganzen nebensächliche Rolle. Kulturelle Beziehungen infolge Handels, Kriege, Kopfjagden, vereinzelt auch durch Heiraten, unter sich, zu der Inlandbevölkerung und zu andern Küsten- und Inselstämmen bestehen seit alter Zeit; doch waren diese Stämme, obwohl einander benachbart, bis zur Ankunft der Weißen unter sich nichts weniger als befreundet. Etwa seit der letzten Jahrhundertwende haben alle diese Leute engeren und engsten Kontakt mit den Weißen, wodurch das ursprüngliche Kulturbild in der jetzt lebenden Generation nicht unwesentlich verändert ist. Jedoch ist diese europäische Beeinflussung, deren Höhepunkt in der Zwischenkriegszeit liegt, noch nicht alt genug, um mehrals einen Oberflächenanstrich zu liefern, unter dem noch sehr viel Altes weiterlebt. Überall findet man noch 


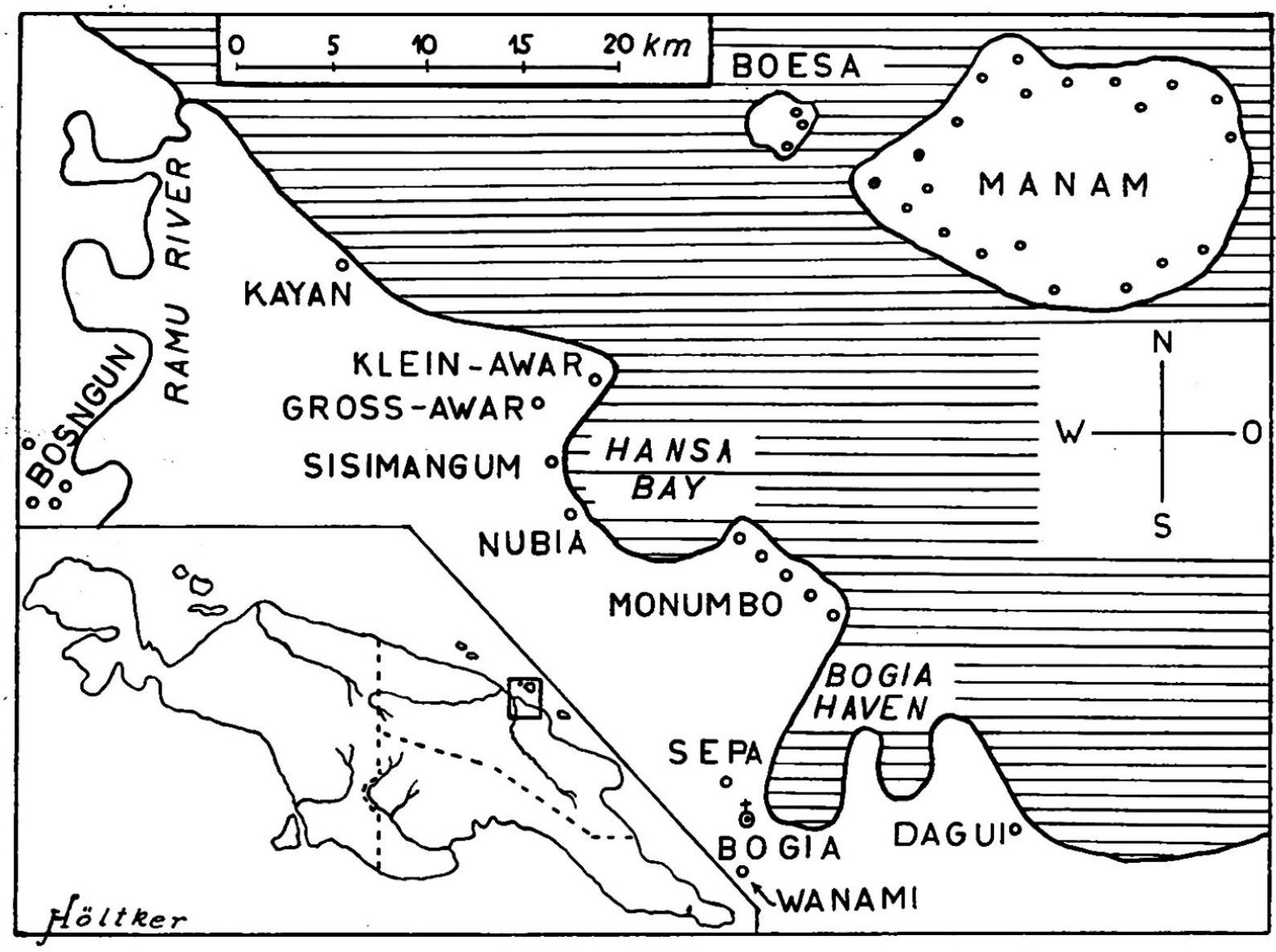

Siedlungen und Stämme des Bogiabezirks, Neuguinea

Gewährsleute aus der fremdenlosen Zeit, jedenfalls bei den Nubia-Awar und Manam, während die Monumbo und Sepa-Wanami den beiden Missionspionieren und bekannten Forschermissionaren F. VormanN, SVD., und J. Schebesta, SVD., die Rettung alten, wertvollen Kulturgutes für die jetzige Generation und für die Wissenschaft verdanken.

\section{Die Manam und Boesa}

Die Vulkaninsel Manan liegt etwa $15 \mathrm{~km}$ östlich vor der Hansabucht (dem Wohngebiet der Nubia-Awar) und dem Potsdamhafen (dem Wohngebiet der Monumbo). Die Insel zählte 1936 nach amtlicher Zählung 4077 Bewohner, die alle, zur gleichen Kultur gehörig, dieselbe melanesische Manam-Sprache (mit ganz geringfügigen dialektischen Unterschieden) sprechen. Die Meistzahl der Dorfsiedlungen liegt unmittelbar oder doch nahe am Strande rund um die Insel. Nur einige wenige Dorfflecken sind etwas mehr bergaufwärts angelegt. Die Insel ist ein einziger Bergkegel mit ständig tätigem Vulkan. Zum Manam-Volk gehören noch sprachlich und kulturell die Leute auf der kleinen Insel Boesa (242 Bewohner) und auf der Vulkaninsel Biem (300 Bewohner). Die nachfolgenden Angaben beschränken sich auf Manam und Boesa, da ich auf Biem nicht lange genug verweilen konnte'.

1 Uber meine Forschungen auf Manam und den Nachbarinseln berichtet kurz ein erster Vorbericht; G. HöLtKER: Neue ethnologische und anthropologische Forschungen auf Manam und seinen Nachbarinseln (Anthropos, 32, 1937, S. 647-649). Später war ich noch einmal längere Zeit auf Manam. Uber die Vulkantätigkeit des etwa $4700 \mathrm{ft}$. hohen Manam vgl. G. HöLTKER: Meine Beobachtungen über die Vulkantätigkeit in Kaiser-Wilhelms-Land, Neuguinea, 1936-1939 (Zeitschrift der Deutschen Geologischen Gesellschaft, 94, 1942, S. 550-560, mit Abbildungen und Kartenskizze). 
Die Ortung erfolgt auf Manam (und Boesa) niemals nach der Sonne oder nach den Himmelsgegenden, sondern ausschließlich nach dem Meer rings um die Insel, wo immer auf Manam der Eingeborne auch sein mag. Das ergibt sich eindeutig aus den entsprechenden Ausdrücken der Manam-Sprache:

Die Richtung auf das Meer hin heißt:

als Substantiv: ilau;

als Verbalsubstantiv (allerdings wenig gebräuchlich): otianga;

als Verbum: oti, d. h. «seewärts gehen".

Steht zum Beispiel auf der Nordseite des Manam ein Eingeborner, und will er an den Strand gehen, so geht er nach der in unserm eigenen Weltbild gebräuchlichen Terminologie nach Norden; für ihn aber ist in diesem Falle der Norden ilau, d. h. die Gegend, wo das Meer ist. Dasselbe gilt, wenn er auf der Ostseite nach Osten, auf der Südseite nach Süden, auf der Westseite nach Westen geht. Alle diese Gegenden sind in diesen Fällen für ihn ilau, und immer gebraucht er dasselbe Verbum oti («auf das Meer zugehen») und das gleiche Verbalsubstantiv otianga (das "Seewärtsgehen»). Weiter werden dieselben Ausdrücke aber auch gebraucht, wenn man von irgendeiner Stelle am Strande aus auf die See hinausfahren will; auch die hohe See rund um Manam ist ilau.

Die Richtung auf die Insel zu und landeinwärts heißt:

als Substantiv: auta;

als Verbalsubstantiv (wenig gebräuchlich): oroanga;

als Verbum: oro, d. h. "landwärts gehen».

Sind beispielsweise die Manam-Leute mit ihren großen, seetüchtigen Kanus auf Handelsreisen gewesen und nähern sich nun bei der Heimkehr wieder ihrer Heimatinsel, so rudern und segeln sie in die Richtung, die auta heißt, ganz gleich, welche Himmelsgegend das ist, und gebrauchen das Verbum oro («auf die Insel zu rudern oder segeln»). Auch der Weg von irgendeiner Stelle am Strande aus landeinwärts führt in die Richtung auta; das dazugehörige Verbum ist immer oro.

In dieser Weise wird die Hauptachse Seewärts - landwärts für jeden beliebigen Punkt auf der Insel festgelegt. Die Querachse dazu bildet der Mensch mit seinen ausgebreiteten Armen. Steht er mit seinem Gesicht zum Meere hin, sieht er also in die Richtung ilau, dann heißt die Ortung zu seiner rechten Hand:

als Substantiv: ata;

als Verbalsubstantiv: irake;

als Verbum: rake, d. h. «nach rechts gehen». •

Die Ortung zu seiner linken Hand heißt in diesem Falle:

als Substantiv: aoa;

als Verbalsubstantiv: ibala;

als Verbum: bala, d. h. «nach links gehen».

Nun aber umgekehrt: steht der Mann am Strand mit dem Rücken zum Meer, mit dem Gesicht landeinwärts gerichtet, sieht er also in die Richtung auta, dann heißt die Ortung zu seiner Rechten nicht etwa ata, sondern aoa, und zu seiner Linken nicht aoa, sondern ata. Daraus ergibt sich, daß weder die Substantive ata und aoa noch auch die entsprechenden Verben rake und bala etwas mit "rechts» und «links» in unserm Sinne zu tun haben können ${ }^{2}$. Diese wie auch alle andern Ausdrücke lassen sich leider nicht

${ }^{2}$ Hier muß ich allerdings auf eine kleine Lücke in meiner Forschung hinweisen, die ich weder damals noch später ganz schließen konnte. Es ist mir nicht gelungen, ganz eindeutig und jeden $Z$ weifel ausschließend festzulegen, ob die Ortung in diesem letzten Falle wirklich überkreuz geht, oder ob nicht doch auch hier «rechts» = ata bzw. rake, und «links» = aoa bzw. bala heißt. Man vergleiche dazu auch die Angaben und gleichen Wörter bei den Monumbo. 
mehr weiter analysieren, so daß man aus der Etymologie keine Schlüsse mehr auf die Grund- und Wurzelbedeutung ziehen kann.

Eine Schwierigkeit könnte man vorbringen: Wenn der Manam-Mann irgendwo auf der Insel mit dem Gesicht zum Meere steht, also in die Richtung ilau sieht, dann hat er hinter sich nicht nur, wie angegeben, die Richtung auta ( "landwärts»), sondern doch auch, quer über die Insel hinweg, wiederum das Meer, also ilau. Das stimmt nach unserm geographischen Weltbild, nicht aber nach dem des Manam-Insulaners. Weil nämlich die Insel ein einziger hoher Vulkanberg und darum querüber unpassierbar ist, rechnen die Manam gar nicht mit dieser abstrakten Möglichkeit; sie müssen, wenn sie von einem Ort auf Manam zu einem andern, etwas weiter entfernten Ort wollen, immer zuerst wieder an die Küste hinab und dann am Strand entlang gehen.

Abschließend seien von Manam noch einige Ausdrücke genannt, die ebenfalls zur Ortung gehören, aber mit den vorhin genannten wohl nichts zu tun haben:

aro bedeutet "vorn", sowohl vorn vor dem eigenen Körper als auch vorn vor irgend etwas, aber in diesem Falle nur räumlich, nicht zeitlich ("vor») vor irgend etwas. Der Ausdruck muri bedeutet "hinten», und zwar ganz als das entsprechende Gegenstück zu dem eben genannten "vorn"; auch in diesem Falle immer nur räumlich gemeint. Weiter gibt es ein Substantiv aoa, das soviel wie "Eingang", "Öffnung», "Mund» usw. bedeutet, und ein anderes, diesem gegenüberstehendes Substantiv kabi mit der Bedeutung "Hintergrund", "Inneres" usw. aoa und kabi sind ein zusammengehöriges Gegensatzwortpaar, so wie auch "vorn» und "hinten». Ob dieses aoa mit dem vorhin genannten aOa ("Richtung nach links») etwas zu tun hat, läßt sich aus dem Manam-Material allein nicht einmal vermutungsweise beantworten.

\section{Die Monumbo}

Die Monumbo wohnen in einigen Küstendörfern am Potsdamhafen, gegenüber der Insel Manam. 1936 zählte der Stamm, der jetzt langsam ausstirbt, noch 209 Individuen. Ihre Sprache ist eine reine, aber schwierige papuanische Sprache (Klassensprache). Sprachverwandt mit den Monumbo sind die Ngaimbom, teils Küstenleute, teils Inländer, in den Dörfern Dagui (55 Bewohner), Mopu (92 Bewohner), Sawawat (116 Bewohner), Lilau (246 Bewohner) und Ambana (218 Bewohner) ${ }^{3}$. In den folgenden Angaben berücksichtige ich aber nur die eigentlichen Monumbo am Potsdamhafen.

Zunächst einige Ausdrücke zur Ortung:

Osten: ikia (Manam: ilau);

Westen: ikorapo (Manam: auta);

Norden: ikasa (Manam: aoa);

Süden: ikusuka (Manam: ata).

Ich gehe nach Norden: angese (Manam: ibala oder ubala);

ich gehe nach Süden: anguande (Manam: urake oder irake);

ich gehe nach Osten: aruanguande (Manam: outi oder oti);

ich gehe nach Westen: aiaro (Manam: uoro oder oro).

Ich komme von Süden: akari (Manam: ubala oder ibala);

ich komme von Norden: akari (Manam: urake oder irake);

ich komme von Osten: asari (Manam: uoro oder oro);

ich komme von Westen: asiakari (Manam: uoti oder oti).

${ }^{3}$ Zur geographischen Lage des Ngaimbom-Gebietes vgl. die Kartenskizze in G. HöLtKer: Verstreute ethnographische Notizen über Neuguinea (Anthropos, 35-36, 1940-1941, S. 44). In die diesem vorliegenden Aufsatz beigegebene Kartenskizze habe ich nur das eine Ngaimbom-Dorf Dagui eingetragen. 
Die in den Klammern beigefügten Ausdrücke aus der Manam-Sprache wurden von den Monumbo selbst angegeben. Dadurch erklären sich die geringen dialektischen Unterschiede zu den von den Manam selbst angegebenen Formen. Die Monumbo sagten aber ausdrücklich, daß ihre eigenen Bezeichnungen mit den entsprechenden Manam-Wörtern gleichbedeutend seien.

Die Monumbo sind von allen Stämmen am längsten und intensivsten mit den Weißen in Berührung gekommen. Die Missionsschule besteht bei ihnen schon seit vierzig Jahren. Alle Bewohner haben eine regelmäßige Schulbildung bekommen.

Heute reden die Monumbo tatsächlich von den vier Kardinalpunkten Osten, Westen, Süden, Norden ${ }^{4}$ und haben in ihrem geographischen Weltbild auch eine entsprechend richtige Vorstellung davon. Diese rein solare Ortung ist aber nichts Ursprüngliches, sondern das Ergebnis der Schulbildung, wie ich nachher noch näher z.eigen werde. In alten Zeiten hatten auch die Monumbo nur eine maritime Ortung.

\section{Die Sepa und Wanami}

Diese beiden kleinen Stämme wohnen unmittelbar am Bogiahafen. 1936 zählten beide zusammen nur noch 194 Köpfe. Vor vier oder fünf Generationen sind die Sepa und Wanami aus irgendeinem Grunde (Vulkanausbruch? kriegerische Handlungen?) von der Insel Manam zum Festland Neuguinea gekommen und haben sich am Bogiahafen angesiedelt. Sie sind also rechte Manam-Leute und sprechen auch einen Dialekt der melanesischen Manam-Sprache. Kulturell sind die beiden Stämme der Sepa und Wanami weitgehend einander gleich, von einzelnen Besonderheiten abgesehen; aber das Bewußtsein, zwei verschiedenen Stämmen anzugehören, blieb doch lebendig. Lange Zeit haben beide Stämme schonungslose Kriege gegeneinander geführt. In Zeiten der Not suchte jeder Stamm lieber Hilfe und Unterstützung bei den umwohnenden Papuanern als beim bluts- und sprachgleichen Nachbarstamm. Erst unter dem Einfluß der Weißen (Regierung und Mission) seit etwa 35-40 Jahren haben sich beide Stämme näher zueinander gefunden, nachdem die Bruderkriege auf hörten. Aus diesen Verhältnissen ergibt sich, daß die Sepa und Wanami in alter Zeit, nachdem sie auf dem Festland Fuß gefaßt hatten, sehr stark durch die umwohnenden Papuaner kulturell beeinflußt wurden und dazu in neuerer Zeit auch von den Weißen durch Schulbildung und täglichen Umgang manches gelernt und angenommen haben.

Zwei Gruppen von Ausdrücken für die Ortung sind bei den Sepa-Wanami zu unterscheiden:

In der ersten Gruppe wären zunächst folgende Ausdrücke zu nennen:

1. otianga (als Verbalsubstantiv): "Osten» und "Das Gehen nach Osten»; oti (als Verbum): «nach Osten gehen».

2. oleanga (als Verbalsubstantiv): «Westen» und «Das Gehen nach Westen»; olo (als Verbum): «nach Westen gehen» ${ }^{5}$.

3. lakonga (als Verbalsubstantiv): «Süden» oder «Norden»; lako (als Verbum): «nach Süden» oder "nach Norden gehen».

4. sopu (als Verbum): "von Süden» oder "von Norden kommen».

Dazu wäre zu bemerken, daß die beiden Verben lako und sopu an sich nur im weiteren Sinne mit Ortung etwas zu tun haben; denn sopu heißt einfach "herkommen»

4 Unter diesen Stichwörtern sind auch die entsprechenden Ausdrücke im Lexikon der MonumboSprache zu finden; F. VormanN, SVD., und W. Scharfenderger, SVD.: Die Monumbo-Sprache (Linguistische Anthropos-Bibliothek, I). Wien 1914. Sub verbis.

${ }^{5}$ Das olo der Sepa-Wanami und das gleichbedeutende oro der Manam sind nur dialektisch verschieden. 
und lako "fortgehen»; das ist dann entweder Süden oder Norden, je nachdem, wo der Sprecher steht. Es könnten die beiden Verben sogar sinngemäß auch für Osten und Westen gebraucht werden, aber bezeichnenderweise finden dann nur die Ausdrücke oti und olo Verwendung.

Den Sepa-Wanami sind heute, so wie den Monumbo, die Begriffe Osten, Westen, Süden, Norden geläufig. $\mathrm{Daß}$ es sich auch in diesem Falle um neuere Einführungen handelt, wird später nachzuweisen sein.

Eine zweite Gruppe von Ausdrücken für die Ortung umschließt die Bezeichnungen für "vorn» und "hinten», im räumlichen Sinne gemeint, wie schon bei den Manam angegeben. Als Verben bedient man sich dafür ebenfalls des schon genannten oti und olo. oti bezieht sich auf alles, was "vorn» ist, also "Mund», "Eingang", "Öffnung", "Zugang" usw. olo ist auf das Dahinterliegende gerichtet, also auf «Inneres», "Hintergrund» usw.

\section{Die Nubia und Awar}

Die Nubia und Awar sind zwei Lokalgruppen des gleichen Stammes, der als solcher keinen besonderen Namen hat. Die Leute selbst nennen sich nur nach ihrer Lokalgruppe Nubia bzw. Awar. Die Nubia wohnen in den beiden Küstendörfern Nubia und Sisimangum, die Awar in Klein-Awar an der Küste und in Groß-Awar, etwa eine Viertelstunde Weges landeinwärts gelegen. Alle vier Dörfer zählten 1936 zusammen 335 Köpfe. Die Nubia-Awar sprechen eine rein papuanische Sprache, die mit der Bosngun-Sprache am Unterlauf des Ramu River nahe verwandt ist. Doch haben die Nubia-Awar mit den Bosngun keine engere Verbindung mehr, nachdem sich die beiden Stämme in alter Zeit weit voneinander entfernt angesiedelt haben. Beide waren und sind bis in dieses Jahrhundert hinein weit und breit gefürchtete Kopfjäger gewesen. Die nachfolgenden Angaben beziehen sich nur auf die Nubia-Awar und lassen die Bosngun aus dem Spiel.

Die Ortung der Nubia-Awar ist sehr interessant und lehrreich. Auch sie orientieren sich nicht nach dem Stand der Sonne, sondern nach den konkreten örtlichen Gegebenheiten. Zum Verständnis müssen wir zuerst zwei Begriffe etwas abklären: mbang und ndiet. mbang heißt zunächst "Fußspur», wird aber in der Regel nur im übertragenen Sinne für irgendeine Richtung verwendet. Die Richtung selbst kann an sich ganz beliebig sein und wird nach irgendeinem festliegenden Punkte (Dorf, Berg, Lagune, Baum, Busch, Meer usw.) näher bestimmt. Die Richtung nach dem Küstendorf Kayan $z$. B., das von Nubia aus nordwestlich liegt und in der Nubia-Sprache Kain heißt, wird Kain-mbang ("Die Richtung nach Kayan») genannt. Die Monumbo-Dörfer, in der Nubia-Sprache Manggér genannt, liegen von Nubia-Awar aus in südöstlicher Richtung. Diese wird darum Manggér-mbang ("Die Richtung nach Monumbo») genannt. So können zwei beliebige Punkte im Gelände durch Hin- und Herbeziehung mittels mbang («Richtung») miteinander verbunden werden. Das ist dann fallweise gleichsam eine festgelegte Achse. Diese Achse teilt dann selbstredend das Gelände in $z w e i$ Teile oder Hälften; diese beiden Hälften werden immer ndiet genannt. ndiet heißt eigentlich nur «Teil, Hälfte, Stück». Eine nähere Bestimmung gibt dann an, welcher Teil (ndiet) gemeint ist. In einer graphischen Darstellung müßte demnach die ndietAchse immer senkrecht zur mbang-Achse stehen.

Die nähere Bestimmung einer Geländehälfte, die man gerade meint, kann auch mit "rechte Hälfte» und "linke Hälfte» erfolgen, eine Bezeichnung also, die vom menschlichen Körper genommen ist. Es können aber auch bestimmte geographische Punkte (z. B. ein Dorf) die nähere Bezeichnung abgeben. Unter diesen dann selbstverständlich auch das Meer, und dieser konkrete Einzelfall interessiert uns hier besonders. Nehmen wir als Beispiel die Achse Kayan-Monumbo, also eine NW-SO-Achse (Kain- 
mbang-Manggérmbang), dann weist die senkrecht daraufstehende ndiet-Achse nach Osten hin auf das Meer, nach Westen hin in den Busch (Urwald). In diesem Falle wird die Meerseite ting-ndiet, die Buschseite ngün-ndiet genannt. Diese Ausdrücke sagen uns, daß die Nubia-Awar auch die geographische Ortung nach "vorn» und "hinten" kennen; denn ting heißt "Mund», "Mündung", "Eingang", "Rand» (am Tontopf, Bambusrohr, Schnurtasche usw.), während ngün "Hintergrund», "Inneres» (im Haus, Schlafsack, Bambusrohr, Netz usw.), "Boden» (im Tontopf) usw. heißt. Der Sinn ist klar: in diesem konkreten Falle liegt an der Achse Kayan-Monumbo die "offene, vordere» Seite nach Osten, zum Meer hin, die "hintere, innere» Seite nach Westen, zum Busch hin. Mit einer Ortung nach dem Sonnenstand hat das keine Beziehung.

Im weitern haben die Nubia-Awar die beiden Verben mbuere und ndziere, die uns interessante Einblicke in die vom Meer hergenommene Ortung gestatten. Beide Verben beziehen sich zunächst auf die Gezeiten. mbuere (und dafür auch das Verbum moandane) heißt zunächst: "die Flut kommt», und ndziere: "die Flut geht zurück», "es ist Ebbezeit». Im übertragenen Sinne heißt mbuere (bzw. moandane) auch: "landeinwärts gehen", "stromaufwärts gehen», vom Meer aus «an den Strand kommen» usw.; während umgekehrt ndziere dann soviel heißt wie "stromabwärts gehen", "vom Busch an den Strand kommen", "auf die hohe See hinausfahren» usw. Dabei wird dann häufig die eigentliche Tätigkeit der Bewegung durch ein besonderes Verbum wie «laufen», «gehen», «rudern», «schwimmen» u. ä. ausgedrückt, dem sich zur Bezeichnung der Richtung "landwärts» bzw. "seewärts» das Verbum mbuere (oder: moandane) bzw. ndziere als selbständiges Verbum (wenn auch, grammatikalisch gesprochen, im adverbialen Sinne) zugesellt. Jedenfalls ist das die Regel. Doch sei noch ausdrücklich gesagt, daß diese Verben mbuere (bzw. moandane) und ndziere auch durchaus allein und für sich stehen und selbst die verschiedenen Arten der Bewegung wiedergeben können. Der Sinn ist also: man tut genau das, was das Meer im gegebenen Falle tun würde, d. h. seewärts oder landwärts gehen.

Schließlich muß noch eine eigenartige Ortung genannt werden, die uns zeigt, wie stark sich die Nubia-Awar durch die konkreten geographischen Verhältnisse bestimmen lassen. Die Dörfer der Nubia-Awar liegen unmittelbar an der im letzten Kriege so oft genannten Hansabay, und zwar liegt Klein-Awar fast genau nördlich von Nubia. Beide Dörfer haben im Osten die große, weite Hansabucht und das offene Meer. Der erste und allgemeine Eindruck, den das Meer bietet, läßt uns ohne weiteres glauben, die Flut erreiche die beiden Dörfer um die gleiche Zeit. Das ist aber nicht der Fall, wie genaue Beobachtungen, in denen die Naturvölker ja Meister sind, zeigen; besonders an dem Treibholz, das die Ebbe ablagert und die Flut wieder mitnimmt, läßt sich das ablesen. Die geographische Eigenart der Bucht bringt es mit sich, daß die Flut um kurze Zeitspannen jeweils früher in Nubia ist als in Klein-Awar, und umgekehrt, daß bei der Ebbe Klein-Awar früher frei wird als Nubia. Die Eingeborenen sagen: Das Meer geht während der Flut (mbuere) von Nubia nach Klein-Awar und bei der Ebbe (ndziere) von Klein-Awar nach Nubia. Darum sagt man von einem Mann, der von Nubia nach Klein-Awar geht oder umgekehrt, er gehe "wie das Meer", und gebraucht das Verbum mbuere, wenn er von Nubia nach Awar, und das Verbum ndziere, wenn er den Weg in umgekehrter Richtung geht. Tatsächlich geht er aber in diesem Falle in südnördlicher bzw. nordsüdlicher Richtung. Es gilt diese Ortung nur an der Bucht selber; sie kommt darum fallweise auch mit der mehr allgemeineren Ortung «landwärts-seewärts» in Kollision. Ein Beispiel: Wenn ein Nubia-Mann in seinem Dorfe sagt: nggo mbuere («ich gehe wie das Meer»), so kann das ebensogut bedeuten: «Ich gehe nach KleinAwar» (also in nördlicher Richtung!) wie auch: "Ich gehe landeinwärts in den Busch» (also in westlicher Richtung!). Das Gegenbeispiel: Ein Awar-Mann in seinem Dorfe sagt: nggo ndziere; das kann heißen: «Ich gehe nach Nubia» (nach Süden!) oder: «Ich gehe auf die See hinaus" (nach Osten!). 
Zum Abschluß noch eine kurze Bemerkung: Jeder Feldforscher weiß, wie stark die Primitiven durch die Art der Fragestellung beeinflußt werden können. Auf meine Frage nach dem "Osten» (dem Ort, wo die Sonne morgens zutrst sichtbar wird) und dem «Westen» (dem Ort, wo die Sonne abends verschwindet) hatten die Nubia-Awar sofort die Antwort zur Hand und sagten:

$\begin{array}{lll}\text { rau } & \text { nggöre } & \text { na-mbang. } \\ \text { Die Sonne } & \text { sie erscheint } & \text { diese Richtung. } \\ \text { rau } & \text { rigre } & \text { na-mbang. } \\ \text { Die Sonne } & \text { sie verschwindet } & \text { diese Richtung. }\end{array}$

Erst als ich merkte, daß ich hier einfache Umschreibungen meiner Fragen vor mir hatte, kam ich auf die obengenannten, wirklich bodenständigen Ortungssysteme. Tatsächlich werden denn auch im täglichen Leben der Nubia-Awar diese beiden Umschreibungen gar nicht gebraucht. Sie waren für mich eigens zurechtgemacht worden, um auf meine Fragen eine Antwort zu geben.

\section{ZUSAMMENFASSUNG UND ERGÄNZUNG}

Alle vier hier behandelten Stämme kennen keine Ortung im Raum nach den Sonnenständen. (Eine solare Ortung in der Zeit ist selbstverständlich auch diesen Stämmen bekannt. Das steht hier nicht zur Diskussion.)

Diese vier Stämme bieten uns ein lehrreiches Beispiel; denn wir haben hier zwei Extreme (einerseits die reine maritime Ortung bei den melanesischen Manam, andererseits die Ortung vorwiegend nach lokalen geographischen Verhältnissen bei den papuanischen Nubia-Awar) und je zwei Mischformen, das sind auf der einen Seite die Verschmelzung der alten maritimen mit der rezenten solaren Ortung bei den melanesischen Sepa-Wanami und den papuanischen Monumbo, auf der anderen Seite bei den papuanischen Nubia-Awar und melanesischen Sepa-Wanami eine Verquickung der maritimen Ortungselemente mit einem neuen Element, das wir kurz mit dem Sigle «vornhinten» stempeln könnten.

Zur Erklärung des Doppelbegriffes «vorn - hinten» müssen noch einige Tatsachen erwähnt werden. Bei den meisten, wenn nicht gar bei allen papuanischen Stämmen des Bogia-Hinterlandes hat eine gewisse Zweiteilung eine bedeutende Rolle zu spielen. Die Dörfer liegen zumeist auf Hügelkuppen, die in der Regel nur von einer oder zwei Seiten aus leicht erreicht werden können. Bei manchen Stämmen, z. B. bei den Awarken, werden diese Zugänge noch mit Bambuspalisaden versperrt; nur ein kleiner, torartiger Eingang gibt den Zugang ins Dorf frei ${ }^{6}$. Die besondere Bedeutung, die dem Dorfeingang zukommt, stellt ihn in einen gewissen Gegensatz zum Dorfinnern. Ähnlich ist es auch bei den Häusern. Vorn am Eingang des Hauses oder auf der Veranda der Pfahlbauten spielt sich das Leben am Tage ab; dort sitzt, raucht und schwatzt man, dort kocht die Frau am Herdfeuer, dort halten sich Besucher und Fremde auf, während das dunkle Innere des Hauses dem intimeren Familienleben, der nächtlichen Ruhe, dem Aufbewahren von Schmuck- und Wertgegenständen dient. Das tiefe Innere eines Hauses wird kaum einmal einem Besucher zugänglich gemacht. Bei den Männer- und Geisterhäusern ist es nicht anders. Auch dort ist der Gegensatz zwischen "Eingang" und "Inneres». Bei den mit einem hohen Zaun umgebenen Gärten ist es ebenso. Den besonderen Einstieg in den Garten kann man fallweise noch durch Fußangeln, Zaubermittel usw.

- J. Schebesta, SVD.: Ein paar erste Notizen über die Awarken in Neuguinea (Anthropos, 35-36, $1940-1941$, S. 978). 
schützen. Merkwürdigerweise hat nun dieser bewußt gewordene Gegensatz im materiellen Kulturbereich auch auf die Soziologie abgefärbt. Oder war es umgekehrt? Der ganze Stamm wird nämlich in zwei große Klassen aufgeteilt, die aber nicht (oder nicht mehr) mit den ethnologisch bekannten beiden exogamen Heiratsklassen identisch sind; denn diese Klassen brauchen nicht rigoros exogam zu sein. Die Zugehörigkeit eines Individuums zu der einen oder andern Klasse wird häufig nicht durch Geburt und Vererbung, sondern ganz einfach durch den Willen der Alten bestimmt. Die Zugehörigkeit kann auch aus bestimmten Gründen von der einen zur andern Klasse überwechseln. Die Benennungen der Klassen erfolgt nach verschiedenen gegensätzlichen Wortpaaren. Dahin gehören nun auch die Begriffe "vorn" und "hinten». Es gibt in den Dörfern "vordere Leute» und «hintere Leute». Ursprünglich mögen die "vorderen Leute» vorn am Eingang des Dorfes gewohnt haben, heute ist das nicht mehr die Regel. Die Begriffe sind jetzt fast zu rein soziologischen Bezeichnungen geworden. Natürlich weiß jeder im Dorf, zu welcher Klasse er gehört. Praktische Auswirkungen hat das zum Beispiel aber noch, wenn die Dorfbewohner etwa zu einem Tanzfest in ein anderes Dorf zu Besuch gehen. Dann lagern sich die Mitglieder der "vorderen" Klasse mehr am Eingang des fremden Dorfplatzes, die Angehörigen der "hinteren» Klasse mehr mitten auf dem Dorfplatz. Solche Gelegenheiten einer praktischen Bedeutung gibt es noch mehr. Im übrigen aber habe ich keine Wertgradunterschiede oder Vorrechte zwischen den beiden Klassen feststellen können. Auch müssen die verschiedenen Arten der Zweiklassen in einem Stamm nicht identisch miteinander sein. Alle Stämme zeigen eine auffällige Vorliebe für eine gegensätzliche Dichotomie in den Aufteilungen und Benennungen. Auf die schwierige Frage, ob die wirtschaftliche oder soziologische Dichotomie lokal die erste war, kann hier nicht weiter eingegangen werden.

Aus dieser Dichotomie "vorn - hinten» wird uns die Ortung der Sepa-Wanami verständlich. Für sie liegt der offene Eingang zu ihren Dörfern im Osten, nämlich am Bogiahafen. Darum das gleiche Verbum oti für zwei Bezeichnungen: «nach Osten gehen» und "zum Eingang gehen». Aus dem gleichen Grunde bedeutet das Verbum olo zweierlei: "nach Westen gehen" und «in das Innere gehen». Diese, eben hier lokal zutreffende Identifizierung wird eine Übernahme aus der Dichotomiepraxis der Hinterlandstämme sein; denn die Manam, zu denen die Sepa-Wanami ursprünglich gehörten, kennen diese doppelte Bedeutung der gleichen Verben nicht, weil es lokal nicht mehr stimmt?

Auch im Wortschatz der Monumbo fand ich die Ausdrücke "vorn — hinten» in der angegebenen Nebenbedeutung nicht, obwohl auch für sie der offene Meereszugang zu ihren Dörfern im Osten, der Inlandurwald im Westen liegt. Das mag sich aus den geographischen Eigenarten erklären: Die Monumbo-Dörfer liegen gewissermaßen mit ihrer ganze Breitseite zum offenen Meer hin, während die Sepa-Wanami nur den kleinen Zugang Bogiahafen haben, der eher die Vorstellung von einem "Eingang» zuläßt. Dazu kommt: Die Monumbodörfer sind auf einem sehr schmalen Küstenstreifen gelegen, der im Westen durch die ziemlich hohen Monumbo-Berge zum Inland hin abgeriegelt wird. Die Monumbo hatten darum mit den Inländern nicht den intensiven und ständigen Kontakt wie die Sepa-Wanami.

Anders ist es bei den Nubia-Awar, deren Dörfer zwar auch am offenen Meer liegen, die aber ebenso auch zum Inland hin leicht gangbare Wege haben. Wenn auch die Dichotomie "vorn - hinten" bei ihnen nur eine nebensächliche Rolle spielt, so ist sie immerhin sogar im Ausdruck (Meerseite = "Mundseite»; Buschseite = «Inneres») bekannt und hat auch dort unverkennbar eine gewisse Beziehung zur maritimen Ortung.

"Wohl haben auch die Manam die Ortsbezeichnungen "vorn - hinten", "Eingang - Inneres» (welches Volk hätte sie nicht?), aber ohne Beziehung zur maritimen Ortung. 
Auch die Sepa-Wanami und Monumbo hatten ursprünglich nur eine maritime Ortung. Die heutige Kenntnis der vier Kardinalpunkte Osten, Westen, Süden, Norden ist noch sehr jung. Der Beweis dafür ist nicht schwer. Die Monumbo haben uns das durch die Beifügung der gleichbedeutenden Manam-Ausdrücke besonders leicht gemacht. Was die Monumbo heute «Osten» nennen, ist für die Manam ilau («seewärts»); der «Westen» der Monumbo ist für die Manam auta («landwärts»). Das stimmt nämlich für die Monumbo in der Wirklichkeit ausgezeichnet: sie haben im Osten das Meer, im Westen das Inland. Die ursprüngliche Bedeutung der Monumbo-Wörter ikia (angeblich "Osten») ist darum "seewärts", und ikorapo (angeblich "Westen») ist "landwärts». Genau so ist es bei den andern Ortungsausdrücken der Monumbo. Die Sepa-Wanami geben uns durch ihre eigene Sprache, die ja eine Manam-Sprache ist, den Beweis für die ursprüngliche maritime Ortung. oti heißt bei den Sepa-Wanami "nach Osten gehen», bei den Manam "seewärts gehen», olo (= oro) bei den SepaWanami «nach Westen gehen», bei den Manam «landwärts gehen». Auch hier erklärt sich die Sache vollständig aus der geographischen Wirklichkeit: die Sepa-Wanami haben das Meer im "Osten», das Inland im "Westen». Für die Monumbo wie für die Sepa-Wanami war es deshalb auch sehr leicht, aus den konkretenVerhältnissen heraus mit ihren alten Vorstellungen von «seewärts — landwärts» die neuen europäischen Begriffe "Osten - Westen» zu verbinden; denn für sie war beides ein und dasselbe.

Alle diese Stämme haben als Grundform der Ortung zunächst eine festliegende Achse. Bei den Manam, Sepa-Wanami und Monumbo heißt sie «Meer-Land» und liegt von Natur aus fest. Bei den Nubia-Awar heißt sie "Ort zu Ort» und kann durch den Menschen beliebig im Gelände festgelegt werden; aber für eine gewünschte Ortung im gegebenen Falle liegt auch sie dann fest. Alle Stämme sind aber mit dieser einen Ortungsachse nicht zufrieden. Sie konstruieren dazu in Gedanken die kreuzende Senkrechte, benennen diese aber stammweise ganz verschieden und zum Teil mit den eigentlich doch wenig sagenden Ausdrücken "wohin - woher» (Sepa-Wanami). Man darf daraus wohl schließen, daß diese Stämme ursprünglich nur die eine Ortungsachse ("Meer-Land», "Ort zu Ort») hatten, dagegen die Senkrechte dazu aus späteren Bedürfnissen entstand und jünger sein muß.

\section{AUSWERTUNG}

Das hier vorgelegte Material sollte nun eigentlich nach verschiedenen Seiten hin ausgewertet werden. So war es auch zuerst meine Absicht. Der Plan läßt sich aber vorläufig noch nicht mit der erforderlichen Ausführlichkeit verwirklichen, nicht nur deshalb, weil die übrige ethnographische Literatur von andern Völkern sich zumeist über diese Dinge ausschweigt. Viele Feldforscher scheinen auf die interessanten Fragen um das Ortungsthema noch nicht aufmerksam geworden zu sein.

Die volle Auswertung würde zudem den Raum, der diesem Aufsatz redaktionell eingeräumt werden konnte, beträchtlich überschreiten. Die "Raumnot» zwingt zur Kürze. Eine spätere Studie mag hier zur gegebenen Zeit ergänzend eingreifen. Hier kann ich nur dies und das eben andeuten.

Beispielsweise bringt das vorgelegte Material kaum einen nennenswerten neuen Beitrag zur Beantwortung der viel diskutierten Frage nach dem Grad und der Intensität des Orientierungsvermögens bei den Naturvölkern. Daß ein Orientierungsvermögen für einen größeren Raum den Eingebornen, allgemein gesprochen, überhaupt fehle, $\mathrm{da} ß$ sie sich vielmehr nur nach den unmittelbar unter den Augen liegenden Wegemarken (etwa geknickte Zweige, kleine Steinhaufen, merkwürdig geformte Bäume usw.) ${ }^{8}$

8 Damit will ich die praktische Bedeutung dieser Wegemarken, die auch in Neuguinea gang und gäbe sind, nicht verkleinern, ebensowenig, wie wir unsere Wegweiser im Gelände missen möéhten. 
zurechtfinden könnten, wird im Ernst heute niemand mehr behaupten wollen. Jedoch gehen die Meinungen der Forscher über die graduelle Fähigkeit und Ausbildung dieses Vermögens noch beträchtlich auseinander. Grundsätzlich wird man R. Allrer recht geben können, wenn er sagt: "Le primitif ne s'oriente pas d'une façon différente de celle du civilisé 9 . Das gilt qua natura des Orientierungsvermögens; denn die graduellen Unterschiede, die tatsächlich bei Primitiven wie bei Zivilisierten beobachtet werden, erklären sich genügend, wenn man die modifizierenden äußeren Faktoren, wie Naturbeobachtung, Schärfe der Sinne, lebenswichtige Bedürfnisse, wirtschaftliche Umstände (ob Schiffer-, Jäger- oder Bauernvolk) u. a., entsprechend mit in Rechnung stellt.

Auf einen dieser äußeren Faktoren macht unser Material aufmerksam, nämlich auf die einzigartige wirtschaftliche Bedeutung des Meeres für die erwähnten Stämme. Alle sind Anwohner des Meeres. Das Meer ist ihnen neben dem Ackerbau das wichtigste Lebenselement für Fischfang und Handelsreisen, als Salzquelle usw. Für den Inländer, selbst wenn er von seinen Bergen aus das große Wasser sehen kann, hat das Meer nicht diese Bedeutung; man "orientiert» sich darum dort auch nicht nach dem Meer, sondern nach andern örtlichen Gegebenheiten, etwa nach dem Manam, dessen rauchender Gipfel weit landeinwärts zu sehen ist.

\section{Vergleich mit andern Stämmen}

Nach diesen mehr allgemeineren Bemerkungen drängt sich gleich die Frage auf: Sind ähnliche Ortungen nach dem Meer und Wasserlauf auch bei andern Völkern der näheren und weiteren Umgebung bekannt? Leider versagt da, wie schon gesagt, die Literatur fast ganz. Die besten Angaben darüber stammen aus Westceram. A. E. JENSEN hat darüber ausführlich berichtet ${ }^{10}$. In der Sprache der Wemale Westcerams fehlen die Wörter für unsere Himmelsrichtungen. Wohl haben die Küstendörfer Bezeichnungen für die Richtungen nach Sonnenauf- und -untergang. Den Bergdörfern aber fehlen diese Bezeichnungen und auch die damit verbundene Ortung. "Diese richtet sich bei den Alfuren nicht nach dem Sonnenaufgang, sondern nach der Himmelsgegend, in der das Meer liegt. Diese Grundrichtung ,dem Meere zu' oder auch ,flußabwärts' heißt lolau oder lowau.» (S. 61.) Den Blick auf das Meer gerichtet, nennt man die Richtung nach rechts lodi, nach links lokai. In den Dörfern südlich des hohen Zentralgebirges, wo man das Meer nur im Süden sieht, haben demnach die gleichen Richtungsbezeichnungen tatsächlich den umgekehrten Sinn wie in den nördlichen Dörfern mit dem Blick auf das Meer im Norden. "Nun ist es aber nicht so, daß es sich bei dieser Orientierung nur um Bezeichnungen für sichtbare Punkte handelt, wobei jedem, der sie benutzt, gegenwärtig ist, daß es sich nicht um eine Himmelsrichtung und überhaupt nicht um eine Richtungsbezeichnung handelt; vielmehr verstehen die Eingebornen unter lowau tatsächlich eine feststehende Richtung, von der sie überzeugt sind, daß es sich in jedem Orte um die gleiche Richtung handelt.» (S. 63.)

Der Unterschied zu meinen Neuguinea-Stämmen ist, trotz überraschender Gleichheiten in der Ortung, doch recht bedeutend. In Neuguinea sind es Küstenstämme, in Westceram ein Bergvolk, "das keine Seefahrt kennt und seit undenklichen Zeiten in seinem jetzigen Raum lebt. Tatsächlich haben die Bergbewohner keinerlei Tradition irgendwelcher Art, die sich auf eine Herkunft von Übersee beziehen». (S. 64.) Daraus folgt, daß JENSENs geistreicher Deutungsversuch aus dem Fehlen "unendlich ferner Punkte» im Weltbild dieser Primitiven für die Westceramesen wohl richtig sein mag, aber doch nicht zur allgemeinen Erklärung genügt.

${ }^{\vartheta}$ R. Allier: Le non-civilisé et nous. Paris 1927. Appendice III, L'instinct d'orientation, S. 302.

10 A. E. JeNSEN : Forschungen in Westceram. Koloniale Völkerkunde. Koloniale Sprachforschung. Koloniale Rassenforschung. Berichte über die Arbeitstagung im Januar 1943 in Leipzig (Beiträge zur Kolonialforschung. Tagungsband I). Berlin 1943, S. $61-65$. 
Ich erinnere mich, daß man von Paparatava (am Mount Varzin) aus, einer bekannten Missionsstation bei den Gunantuna auf der Gazellehalbinsel (New Britain), das Meer wenigstens in drei ganz verschiedenen Richtungen sehen kann, während in den Zwischenräumen der drei Richtungen Berge und Bäume den Blick verstellen. Für das Auge sind es praktisch drei verschiedene Meere. Es wäre nun sehr interessant und methodisch lehrreich, zu wissen, ob und welcher Art in einem solchen konkreten Falle eine Ortung nach dem Meer existiert. Möglicherweise gibt es auf der Gazellehalbinsel eine solche Ortung; denn daß eine alte Richtungsbestimmung "nach oben — nach unten» analog etwa dem "flußaufwärts - -abwärts» bekannt ist, läßt sich sprachlich belegen ${ }^{11}$. Aus bestimmten Wörtern und Mythenmotiven läßt sich sogar für Paparatava selbst eine gewisse Richtungnahme "meerwärts - landwärts» erkennen ${ }^{12}$, wenn auch die allzu dürftigen Angaben darüber noch nicht für Vergleichszwecke ausgewertet werden können.

Die Spuren einer alten geographischen Ortung «flußaufwärts - -abwärts» lassen sich auch bei den Ngadju Dajak in Südborneo erkennen; denn «der Name Ngadju bedeutet Oberländer. Wahrscheinlich handelt es sich um eine Selbstbezeichnung der Bevölkerung im Unterschied zu den Oloh Tambang: die Leute, die an der Mündung eines Flusses wohnen, d. h. die mohammedanischen Malaien, und den Ot Danum: die Leute, die das Quellgebiet bewohnen ${ }^{13}$. Diese Ngadju Dajak haben sogar eine Verbindung zwischen der solaren und maritimen Ortung geschaffen, indem die vier kosmischen Punkte: Sonnenuntergang-Flußabwärts-Sonnenaufgang-Flußaufwärts (in dieser Reihenfolge!) im Ritual der Opferhandlungen eine bedeutende Rolle spielen ${ }^{14}$. Damit berühren wir schon das religiöse Weltbild.

\section{Das religiös-mythische Weltbild}

JENSEN hat den Nachweis erbracht, daß die maritime Ortung bei den Westceramesen als Teil eines bestimmten religiös-mythischen Weltbildes aufzufassen ist (S. 64-65). Die von ihm beigebrachten Belege lassen darüber in diesem konkreten Falle keinen Zweifel. Sie finden außerdem noch eine Stütze in dem, was uns H. SCHärER, wie oben erwähnt, über die Stellung der maritimen Ortung (im besonderen Aspekt «flußaufwärts - -abwärts»!) im religiös-mythischen Weltbild der Ngadju Dajak erzählt. In einer späteren Publikation hat JENSEN dann diese merkwürdige, religiös-mythisch unterbaute Ortung gewissermaßen als typisch für das Weltbild einer bestimmten frühen Kultur hingestellt, wobei er sich besonders auch auf ganz ähnliche Erscheinungen bei den Uitoto, einem Stamm der südamerikanischen Waldindianer, stützte ${ }^{15}$. Die Berechtigung

11 "Nach dem Sprachgebrauch der Gunantuna begibt man sich in einen Wald immer , hinunter', zu einer Siedlung immer , hinauf', wobei es keine Rolle spielt, ob der Wald höher oder tiefer liegt als die Gehöfte.» (H. KROLL: Der Iniet. Zeitschrift für Ethnologie, 69, 1937, S. 205, Anmerkung 1.)

12 «Mit Raravat bezeichnet der Eingeborne jeden höheren Berg im Innern (des Landes), mit Tiratira jeden Berg am Ufer des Meeres» (A. KLeinrrrschen, MSC.: Mythen und Erzählungen eines Melanesierstammes aus Paparatava, Neupommern. Wien-Mödling 1924, S. 31, Anmerkung 3). "To Kabinana schuf die Dörfer, welche in der Richtung zum Meer liegen, To Purgo dagegen die Dörfer, welche landeinwärts liegen.» (KLeintitschen: S. 38, Anmerkung 2.)

${ }^{13}$ H. Schärer: Die Gottesidee der Ngadju Dajak in Süd-Borneo. Leiden 1946, S. 1. Vielleicht dürfen wir wenigstens die Andeutung einer alten, an den Flußlauf sich anlehnende Ortung auch noch in Zentralborneo erkennen. Bei den Kenja Dajak (Zentralborneo) findet nämlich am zweiten Tage des großen Kopfjägerfestes ein zeremonielles Wettrudern statt, in dem jeweils sich ein Kanu der Flußunterlaufanwohner mit dem der Bewohner am Flußoberlauf im Wettrudern mißt. (J. M. Elshout: De KenjaDajaks uit het Apo-Kajangebied. 's-Gravenhage 1926, S. 306.)

14 SCHÄrER: S. $75-76$.

${ }^{15}$ A. E. Jensen: Das Weltbild einer frühen Kultur (Paideuma, 3, 1944, S. 1-83, besonders 50). 
solcher Gedankengänge ist unzweifelhaft, ihre Richtigkeit sogar mit einer gewissen Wahrscheinlichkeit gegeben. Doch bis zu einem wirklich schlüssigen Beweis wird noch viel Feld- und Forscherarbeit nötig sein.

Das Neuguinea-Material bietet dieser Theorie keine Hilfe, wenigstens vorläufig noch nicht. Wohl hat auch bei meinen Stämmen das Meer in den Mythen und Zeremonien, in Glauben und Magie seinen festen Platz, entsprechend der Wichtigkeit und Eindrücklichkeit seiner Erscheinung für primitive Menschen. Aber es sind doch nicht ausgesprochen Ortungsbegriffe, die da mitreden. Möglicherweise lassen sich später, wenn ich mein Material einmal ganz durchgearbeitet habe, gewisse Spuren oder sogar Hinweise dafür finden; aber bis dahin kann man die profane maritime Ortung meiner Neuguinea-Stämme noch nicht als einen Teil oder als Abbild einer irrationalen «Weltanschauung» oder eines bestimmten religiös-mythischen "Weltbildes» ansehen.

\section{Kulturgeschichtliche Stellung}

Das erste Erfordernis, um zu einer richtigen Erkenntnis der kulturgeschichtlichen Stellung der maritimen Ortung zu kommen, wäre eine methodische Zerlegung dieser komplexen Erscheinung in ihre Bestandteile. Soweit es bis jetzt erkennbar ist, müßte man in dem Neuguinea-Material wohl folgende Elemente unterscheiden: 1. Ortung nach dem Meer, 2. Ortung nach dem Flußlauf, 3. Ortung "vorn-hinten", 4. profanes oder religiös-mythisches Weltbild.

Für das Neuguinea-Material scheidet das 4. Element vorläufig aus. Die eventuelle Zusammengehörigkeit von Element 1 und 2 ist zunächst nur für die Nubia-Awar belegbar, bekommt aber immerhin aus Westceram eine gewisse Unterstützung. Die Selbständigkeit von Element 3 scheint auf den ersten Blick ziemlich fraglich zu sein, da es in Neuguinea, nur Manam ausgenommen, sich immer mit der maritimen Ortung zeigt und sogar bei den Gunantuna in Neubritannien wenigstens andeutungsweise spürbar ist $^{16}$, während es in Westceram und Borneo fehlt. Dennoch glaube ich, daß wir es hier mit zwei von Haus aus wesentlich verschiedenen Ortungssystemen zu tun haben: einmal mit der maritimen Großraumortung ( «meerwärts - landwärts», eventuell noch verbunden mit "flußaufwärts - -abwärts») und dann mit der lokalgebundenen Kleinraumortung ("vorn - hinten»).

Wie schon angegeben, ist der Gegensatz "vorn - hinten» nur eine Form der weit verbreiteten Dichotomie, die sich unter den verschiedensten Benennungen gegensätzlicher Begriffspaare bis tief ins Bogia-Hinterland und sogar bis an den mittleren Sepik River hinauf nachweisen läßt. Alle diese Stämme sind aber (von den Sepa-Wanami abgesehen) Nichtmelanesier (Papuaner). Diese Zweiteilungen brauchen nun zwar heute nicht mit einer rigoros geforderten Exogamie verbunden zu sein; aber man hat den Eindruck, daß es ursprünglich so war. Damit erinnert uns diese Dichotomie sofort an die bekannte Aufteilung bestimmter melanesischer Stämme (z. B. der Gunantuna in Neubritannien) in zwei exogame Heiratsklassen ("Zweiklassenkultur»). Bezeichnenderweise sind die echten exogamen Heiratsklassen noch bis hart vor den Toren Neuguineas vorhanden, z. B. auf der Insel Biem, die der Insel Manam benachbart ist. Darum halte ich die Dichotomie bei den Stämmen im Bogiabezirk, obwohl es sich um papuanische Stämme handelt, nicht für ein "papuanisches», sondern für ein «melanesisches», bzw.

16 Den ortungsbegrifflich empfundenen Gegensatz von «vorn - hinten» finde ich bei den Gunantuna durch KLEINTITSCHEN angedeutet: «Bitangunan bedeutet: Anfang der Gehöfte, wo die Gehöfte eines Distriktes beginnen. Im Gegensatz dazu steht Ulangunana = Kopf, Ende der Gehöfte, wo die Gehöfte auf hören.» (KLEINTIrSCHEN: S. 269, Anmerkung 2.) - «Die vabugan-Seite ist die hintere Ecke der Hütte. Der Mann als der stärkere und mutigere schläft beim Eingang. Dann kommt das Wärmefeuer, und jenseits schläft die Frau. Die Frau schläft also nahe bei der vabugan-Seite. " (KLEINTrTSCHEN: S. 477, Anmerkung 2.) 
wenn wir den Begriff noch weiter fassen wollen: für ein «austronesisches» Kulturelement, das die Papuaner übernommen haben. Möglicherweise ist die Zweiteilung "vorn - hinten» nur eine lokale Sonderform dieser Dichotomie gerade für den Bogiabezirk. Die Andeutungen aus Neubritannien würden allerdings auch die Möglichkeit offen lassen, daß spätere Forschungen die Kleinraumortung "vorn-hinten» als ein weiter verbreitetes, vielleicht sogar als ein allgemein-melanesisches Kulturelement erkennen lassen.

$\mathrm{Daß}$ diese Ortung «vorn - hinten» nicht mit der maritimen Großraumortung identisch ist oder auch nur als notwendiger Komplementärteil dazu gehört, dürfte klar sein, auch wenn sie sich in Neuguinea (und Neubritannien?) faktisch damit verbindet. Bei der Ortung "vorn - hinten " haben wir es freilich a u ch mit einer geographischen Ortung zu tun; aber diese ist verbunden mit einem starken soziologischen Element, das an Bedeutung das geographische übertrifft und wahrscheinlich auch zeitlich das primäre sein dürfte. Bei der maritimen Ortung Neuguineas ist dieses soziologische Element nicht vorhanden. Nun glaube ich, daß die maritime Ortung ursprünglich nur Völkern eigentümlich gewesen sein kann, die am Meere wohnen oder denen doch das Meer von ihrem Wohnplatz aus sichtbar und auch irgendwie von Bedeutung ist. Damit schließt sich die große Masse der "Papuaner» als erste Träger dieses Kulturelementes von selber aus. Alles spricht also dafür, daß wir in den Austronesiern die eigentlichen Träger der maritimen Ortung sehen müssen. Das vorgelegte Material aus Neuguinea, Neubritannien, Westceram und Borneo erhärtet diese Auffassung. Weitere und speziellere Zuteilungen müssen späteren Forschungen überlassen bleiben.

Wenn nun auch sowohl die maritime Großraumortung «meerwärts — landwärts» als auch die geographisch-soziologische Kleinraumortung "vorn - hinten» beide als «melanesische» bzw. "austronesische» Kulturelemente angesprochen werden, so folgt daraus methodisch noch nicht, daß sie auch von Haus aus zusammengehören. Ich sehe beide, solange nicht weitere Forschungen zur Hand sind, als ursprünglich getrennte und verschiedenen Kulturschichten angehörende Elemente innerhalb der sogenannten «austronesischen Kulturen» an, deren jede für sich ja bekanntlich selbst schon wieder ein sehr komplexes Gebilde ist.

\section{L'ORIENTATION (ORTUNG) MARITIME CHEZ QUELQUES TRIBUS AU N.-E. DE LA NOUVELLE-GUINÉE}

"Ortung» signifie l'orientation. En Nouvelle-Guinée il y a quelques tribus qui ne s'orientent pas d'après le soleil mais d'après la mer. Chez les tribus Manam, Sepa-Wanami, Monumbo et Nubia-Awar, ce sont leurs langues qui prouvent ce fait. Mais il y a encore d'autres manières de s'orienter étroitement liées avec cette "orientation maritime", par exemple les Sepa-Wanami et les Nubia s'aident de l'orientation d'après les fleuves («en amont» et "en avab») et d'après les lieux géographiques; chez les Sepa-Wanami et les Nubia-Awar, c'est l'orientation selon «devant et derrière» qui est en usage. Cette orientation maritime qui n'a qu'une signification profane forme probablement un élément dans les «cultures austronésiennes».

\section{L'ORIENTAZIONE MARITTIMA PRESSO QUALCHE TRIBÙ NELLA NUOVA- GUINEA NORD-ORIENTALE}

"Ortung》 = orientazione, metodo di orizzontarsi. Nella Nuova-Guinea alcune tribù non si orientano secondo il sole, ma si riferiscono al mare. Ciò vien provato per i Manam, Sepa-Wanami, Monumbo e Nubia-Awar in base alle loro lingue. Con queste orientazioni marittime son congiunti ancora altri modi di orizzontarsi: per i Sepa-Wanami e Monumbo quello secondo la posizione del sole, introdotto dai bianchi, per i Nubia-A war quello determinato dal corso dei fiumi ("verso l'insù», "verso l'ingiù») e quello che si riferisce all'ubicazione geografica, per i Sepa-Wanami e Nubia-Awar l'orientazione "dinanzi-di dietro». Il metodo marittimo per queste tribù non è in relazione con idee di carattere religioso ed appartiene dal punto di vista colturale alle «colture di Austronesia». 